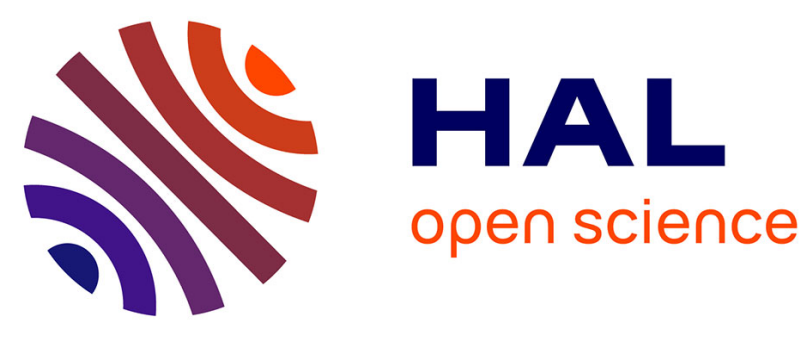

\title{
R2Lab Testbed Evaluation for Wireless Mesh Network Experiments
}

Farzaneh Pakzad, Marius Portmann, Thierry Turletti, Thierry Parmentelat, Mohamed Mahfoudi, Walid Dabbous

\section{- To cite this version:}

Farzaneh Pakzad, Marius Portmann, Thierry Turletti, Thierry Parmentelat, Mohamed Mahfoudi, et al.. R2Lab Testbed Evaluation for Wireless Mesh Network Experiments. ITNAC '18 - International Telecommunication Networks and Applications Conference, Nov 2018, Sydney, Australia. hal-01968766

\section{HAL Id: hal-01968766 https://hal.science/hal-01968766}

Submitted on 3 Jan 2019

HAL is a multi-disciplinary open access archive for the deposit and dissemination of scientific research documents, whether they are published or not. The documents may come from teaching and research institutions in France or abroad, or from public or private research centers.
L'archive ouverte pluridisciplinaire HAL, est destinée au dépôt et à la diffusion de documents scientifiques de niveau recherche, publiés ou non, émanant des établissements d'enseignement et de recherche français ou étrangers, des laboratoires publics ou privés. 


\title{
R2Lab Testbed Evaluation for Wireless Mesh Network Experiments
}

\author{
Farzaneh Pakzad ${ }^{*}$, Marius Portmann ${ }^{\dagger}$, Thierry Turletti ${ }^{\ddagger}$, Thierry Parmentelat ${ }^{\ddagger}$, \\ Mohamed Naoufal Mahfoudi ${ }^{\ddagger}$ and Walid Dabbous ${ }^{\ddagger}$ \\ *Aptira Pty Ltd 43 Marshall Street, Surry Hills, NSW, Australia \\ Email: farzaneh@aptira.com \\ ${ }^{\dagger}$ School of ITEE, The University of Queensland, Brisbane, Australia \\ Email: marius@ieee.org \\ $\ddagger$ Universit Cte dAzur, Inria, Nice, France \\ Email: firstname.lastname@inria.fr
}

\begin{abstract}
We have provided critical evaluations of new potential testbeds for the evaluation of SDN-based WMNs. We evaluated the R2Lab wireless testbed platform at INRIA SophiaAntipolis, France. This testbed has 37 customisable wireless devices in an anechoic chamber for reproducible research in wireless $\mathrm{WiFi}$ and 4G/5G networks. Our work presents the first initial evaluation of the testbed for wireless multi-hop experiments, using traditional WMN routing protocols. Our results demonstrate the potential for SDN experiments. We believe this is an important contribution in its own right, since experimental validation is a key research methodology in this context, and trust in the validity of experimental results is absolutely critical.
\end{abstract}

\section{INTRODUCTION}

R2Lab [1] is a wireless testbed platform located at INRIA ${ }^{1}$, Sophia-Antipolis, France. This platform is part of the FIT federation, which provides an open large-scale, high performance testing infrastructure for performing experiments on systems and applications on wireless and sensor communications. R2Lab is an open testbed located in an anechoic chamber composed of 37 customisable wireless devices, along with USRP (Universal Software Radio Peripheral) [2] nodes and commercial LTE phones for accomplishing reproducible research in $\mathrm{WiFi}$ and $4 \mathrm{G} / 5 \mathrm{G}$ cellular networks.

$\mathrm{R} 2 \mathrm{Lab}$ is equipped with a range of software tools that allow the control of the wireless nodes remotely through a $s s h$ gateway. Each user can reserve the whole testbed for his/her experiment and can take full control of all the wireless devices. The user can run his/her own customised Operating System (OS) on each node in order to perform experiments on customised systems. After loading the OS on a node, it is accessible via $s s h$ with administrative privileges and ready for the user to configure the available resources, such as nodes, USRPs and phones.

The nodes are positioned in a grid layout, as illustrated in Figure 1. Each node is equipped with 3 wired interfaces used for remote power and reset management, control and a data channel dedicated to experimentation. This separation of

\footnotetext{
${ }^{1}$ Institute for Research in Computer Science and Automation.
}

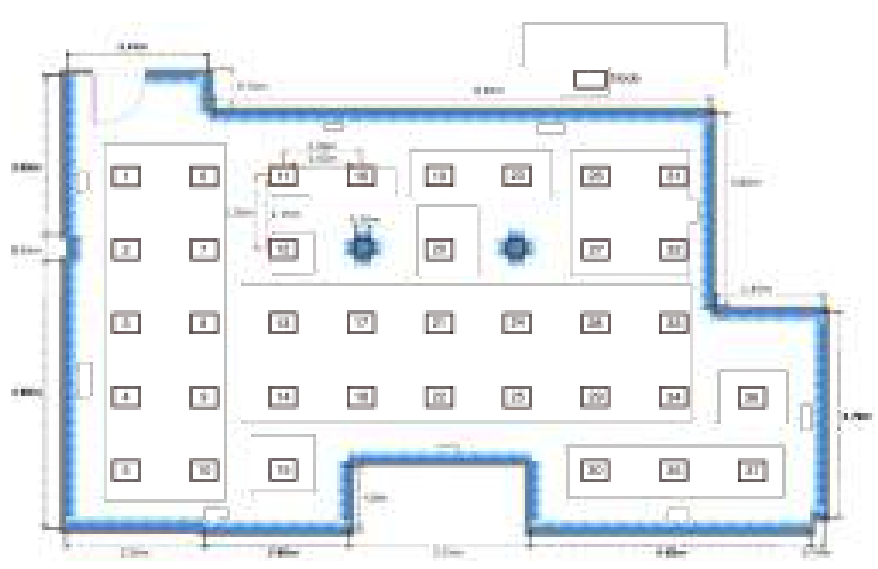

Fig. 1. Ground Plan Layout of Nodes

control and data channels in the testbed makes it a potentially suitable platform for SDN experiments, to be considered in future work.

The launch of the R2Lab platform was in November 2016 [3], and consequently there has been limited use and evaluation of the testbed. In particular, there have been no wireless multi-hop or WMN experiments conducted on the platform, and our work provides the first basic evaluation of the platform for this purpose. In this paper we present the results of our experiments, which consist of a comparison of two widely used WMN routing protocols, i.e. OLSR and BATMAN. Our work, which was conducted during a 5-week research visit at INRIA, validates the R2Lab testbed as a suitable platform for wireless multi-hop experiments, with a great potential for SDN-based WMN experiments.

The rest of the paper is organised as follows. In Section II we discuss key related works, i.e. wireless testbeds. Section III explains our basic experiments to evaluate the suitability of R2Lab for multi-hop wireless networks. Section IV discusses the more complex evaluation of R2Lab by considering Wireless Mesh Networks routing experiments. Finally, Section V provides a summary and conclusions for this work. 


\section{RElAted Work - Wireless Testbeds}

There exists a wide range of work on the design and evaluation of wireless network testbeds and testbed platforms. Here, we give a very brief summary of some of the key works, to provide the context of our own work.

ORBIT is an Open Access Research Testbed for NextGeneration Wireless Networks (ORBIT) [4], [5], founded in 2003 for conducting reproducible wireless experiments. The architecture of ORBIT is a two-tier system consisting of a laboratory-based wireless network emulator and a field trial network. This allows the experimenter to perform the basic experiments on the emulator, which addresses the problem of reproducibility, while at the same time providing the opportunity for the user to evaluate the performance of applications and protocols in real-world networks. The ORBIT lab emulator consists of a large number of static $802.11 \mathrm{x}$ wireless nodes laid out in a grid. This radio grid emulator provides facilities for the user to reproduce wireless network experiments with a specified topology for quantitative evaluation of different protocols and applications. The user can have full access and control to wireless nodes, such as installing their own OS and software packages, rebooting, etc. The user can move to the field trail network in order to support the validation of the results obtained on the emulator. In order to create a multihop network in ORBIT, it is suggested to use MAC address filtering or noise generation. The former cannot omit the contention and interference between multiple senders and the latter is limited in the topologies that can be achieved. ORBIT has a strong experiment control and management capabilities. One of its shortcomings is the lack of control of background noise due to the fact that wireless nodes are not placed in a anechoic chamber.

Emulab [6] is another open access large scale platform for running experiments in computer networking and distributed systems. Emulab has a variety of features, including support for arbitrary network topologies, full control of nodes with arbitrary OS and configuration, and support for both WiFi as well as SDR experiments, using USRPs. Emulab also supports integration with other testbeds such as Planent LAB. As in the case of ORBIT, the Emulab wireless nodes are not isolated from other wireless networks, so the signal from other wireless networks can interfere with any Emulab experiments.

WHYNET (Wireless HYbrid NETwork) [7] is another large-scale hybrid platform for implementing heterogeneous wireless technologies such as WiFi, cellular networks, sensors, etc. It is a hybrid wireless testbed combining hardware testbeds, simulation and emulation. This combination provides the ability to take advantage of the benefits of the different experiment types, i.e. the realism of physical testbeds with the scalability, flexibility and repeatability of simulation and emulation experiments.

In contrast to these above mentioned testbed platforms, R2Lab provides a wireless hardware testbed that can avoid background noise and interference of other nearby networks, via the use of a anechoic chamber, which provides RF isola-

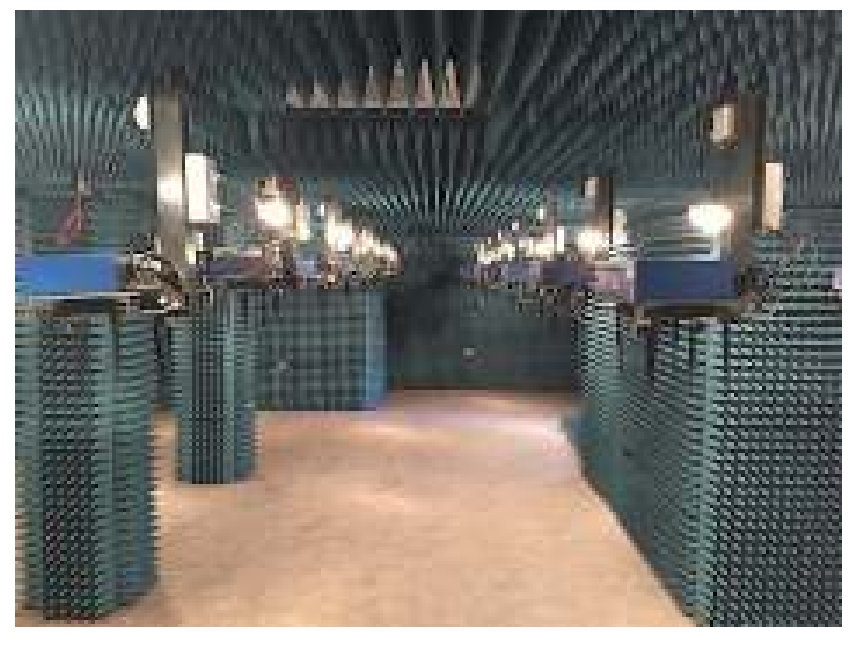

Fig. 2. R2lab Located in an Anechoic Chamber

tion. This is shown in Figure 2.

We will now present our validation of the R2Lab for evaluation of WMN routing protocols.

\section{R2LAB EXPERIMENTS}

For our experiments to evaluate the suitability of R2Lab for wireless multi-hop experiments, we considered two WMN routing protocols, namely Optimised Link State Routing (OLSR) [8] and Better Approach To Mobile Ad hoc Networking (BATMAN) [9].

- OLSR is a link state routing protocol where every node maintains topology information about the entire network. OLSR uses two types of control packets; HELLO and Topology Control (TC) messages. HELLO messages are used by a node to find its one-hop and two-hop neighbours. By discovering these neighbours, each node chooses a set of Multi Point Relays (MPR) based on its one-hop neighbours advising a best route to two-hops neighbours. MPRs aim to reduce the overhead of sending link state information in the whole network. Nodes regularly send TC packets with the information about their neighbourhood and the state of the links between them. MPR nodes forward the TC packets throughout the network. Considering the information in the TC packets, other nodes create a map of the network topology. In OLSR, nodes use a shortest path algorithm to calculate a path towards a destination. OLSR supports both the hop count as well as the ETX routing metric.

- BATMAN is a relatively new proactive routing protocol where each node only maintains information about the best next hop node for each destination, instead of the entire network topology as in the case of OLSR. Consequently, BATMAN reduces the amount of control traffic flowing in the network that can allow lower CPU usage and lower battery consumption of the mesh nodes. The BATMAN routing protocol operates as follows: 


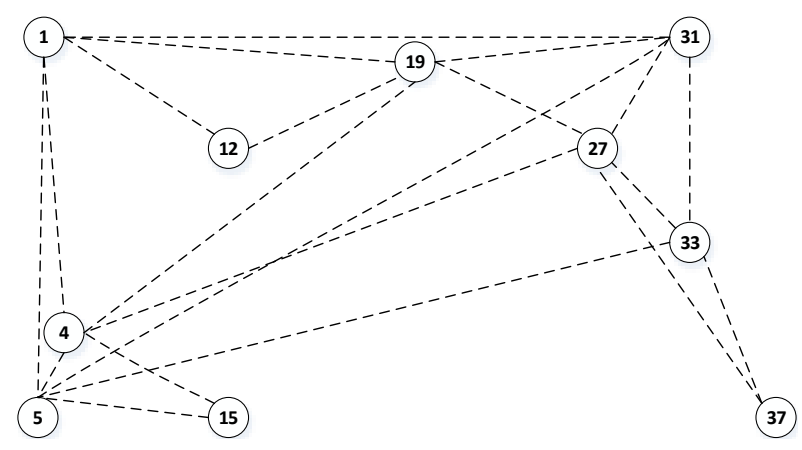

Fig. 3. Our Customised Mesh Topology

- In regular intervals, nodes send an OriGinator Message (OGM) including the originator IP address, forwarding node IP address, Time To Live (TTL), and Sequence Number (SQ), to inform other nodes about the existence of this node.

- Neighbours rebroadcast the OGM messagse and allow other nodes to know about the existence of the OGM originator and so on and so forth. Therefore, OGM messages are flooded across the network.

- BATMAN maintains a table of the number of OGM messages received from each originator and via which one-hop neighbour the messages were received. The one-hop neighbour via which the largest number of OGM messages from a particular originator was received is considered as the best next hop for this destination.

We have evaluated these two WMN routing protocols in terms of Packet Delivery Ratio (PDR) and end-to-end latency in two different network scenarios, one without and one with the addition of interference.

As mentioned before, the R2Lab testbed consists of 37 nodes. Each node includes the state-of-the-art motherboard including an Intel Core i7-2600 processor, 4GB RAM, 240 GB SSD, and is equipped with 2 wireless interfaces, with Atheros $802.1193 \mathrm{xx}$ a/b/g/n and/or Intel 5300 chips, and with 3 antennas each. The WiFi mode used in this experiment was 802.11a. Figure 1 shows the location of the nodes, which are distributed in a roughly $90 \mathrm{~m}^{2}$ area. The distance between nodes is about $1 \mathrm{~m}$ in each direction, with some exceptions near the columns that are supporting the room. We installed Ubuntu Linux 16.04 on each of the nodes.

The first challenge that we had to address was the creation of multi-hop topology. Using the default settings, each node can see every other node, which results in only single-hop paths. By minimising the transmission power to $0 \mathrm{dBm}$ and setting the transmission rate to a fixed $54 \mathrm{Mbps}$, we were able to reduce the transmission range. We then ran ping between every node pair in the topology to find all the active (single-hop) links. We then chose a subset of 10 nodes, which maximises the multihop nature of the network. The resulting topology is shown in Figure 3.

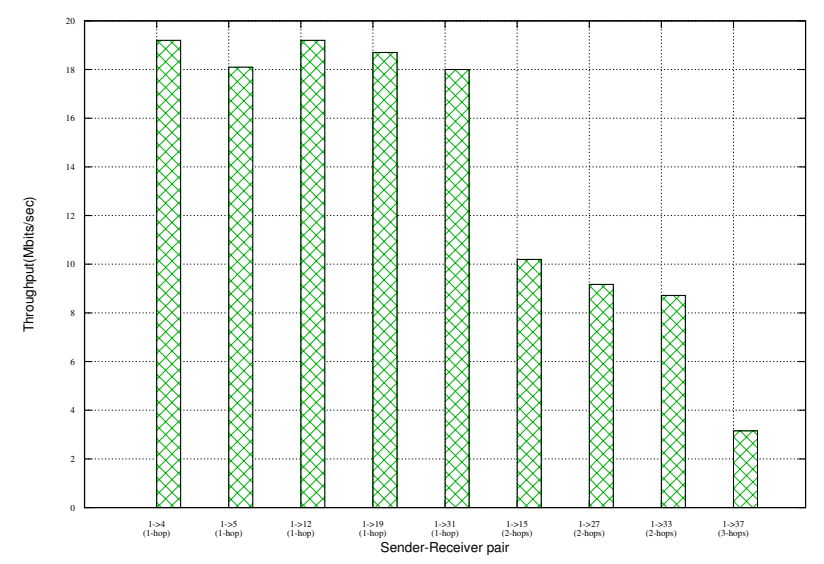

Fig. 4. Throughput vs. Distance over Different Number of Hops

For example, for node 1 to reach node 37 , packets need to traverse at least 3 hops, i.e. via the following path: $1 \rightarrow 19$ $\rightarrow 27 \rightarrow 37$. All our experiments have been conducted using this topology.

We also measured the achievable throughput for different source destination pairs using iperf. We ran iperf with an offered load that guarantees link saturation. We measured the throughput of the network paths between node 1 and every other node. The results of our measurements are shown in Figure 4. We see that for short distances (i.e. one-hop), as shown on the $\mathrm{x}$-axis, the achieved throughput is between 18 and $20 \mathrm{Mbps}$, which is close to the maximum achievable throughput for $54 \mathrm{Mbps} \mathrm{WiFi}$ OFDM. Once the distance between the source and destination increases to two hops, the throughput decreases to roughly half (i.e. around $9 \mathrm{Mbps}$ ), due to the fact that radio interfaces operate in half-duplex mode and cannot receive and send data simultaneously. When the path length reaches three hops, the throughput decreases further, to a value of about $3.15 \mathrm{Mbps}$. This result reflects the expected behavior of wireless multi-hop networks [10], [11], and provides a basic validation of the R2Lab testbed for the use of wireless multi-hop experiments.

In the following we will consider more complex validation experiments, and for this we will consider the WMN routing protocols BATMAN and OLSR, as mentioned earlier.

\section{WMN ROUTING EXPERIMENTS}

In this section, we considered two basic experiment scenarios, a case with no interference and one with interference. We evaluated BATMAN and OLSR in terms of the end-to-end latency and packet delivery ratio for these two scenarios.

In our experiments, we used olsrd version 0.6.6.2 from olsr.org [12] with the default configuration, with the Link Quality (LQ) extensions enabled. This means that the Expected Transmission Count (ETX) was used as the routing metric [13].

For BATMAN, we used batmand version 0.3.2-17 on each node from open-mesh.org [9]. As mentioned before, BATMAN 


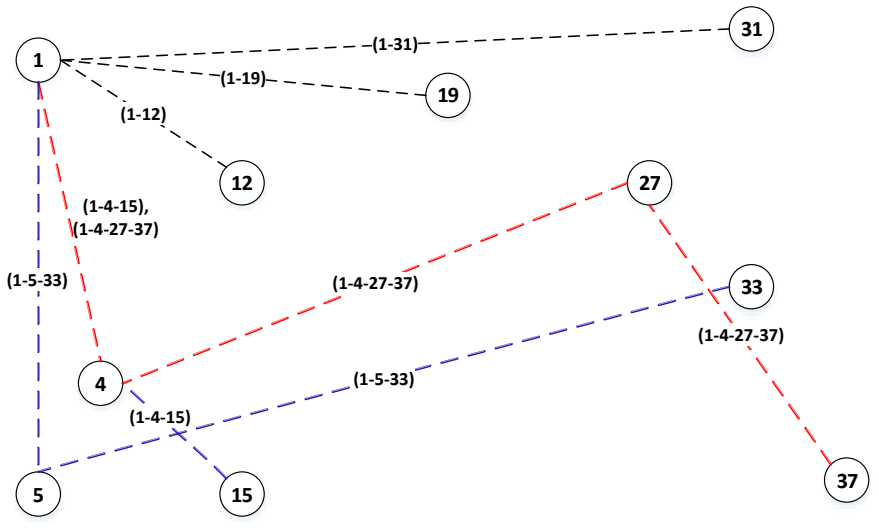

(a) OLSR Routing Protocol

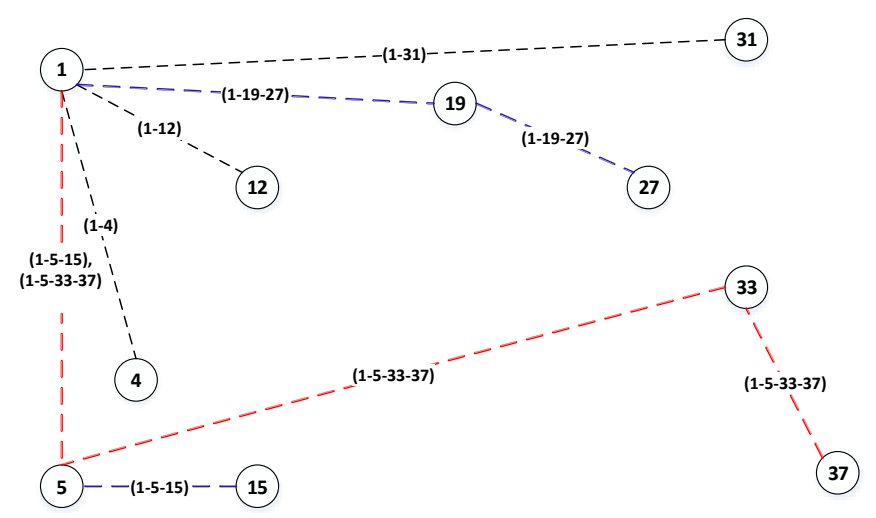

(b) BATMAN Routing Protocol

Fig. 5. Established Route Between Node 1 as Source and Other Nodes as Destinations

also has an awareness of the link/path quality and tries to avoid the routes with lower packet delivery rate.

\section{A. No Interference}

As an initial experiment, we wanted to discover the paths that both BATMAN and OLSR establish in our network topology. For this, we ran each of the two protocols on all nodes (consecutively) and we used the traceroute command to establish all the routes for all source destination node pairs.

As a simple example, we showed how BATMAN and OLSR choose routes between node 1 as a source and all other nodes as destination in the network. As a representative example, Figure 5 shows the established routes between node 1 an all other nodes in the network for OLSR (Figure 5(a)) and BATMAN (Figure 5(b)).

One hop paths are indicated with black dashed lines, blue lines indicate 2-hop paths and red lines 3-hop paths respectively. Since some paths overlap, we added labels for each path, which indicate the sequence of nodes, in order to increase clarity. For example, in Figure 5(a) if node 1 wants to reach node 37 using OLSR, packets will go through nodes 4 and 27. This has been shown with the label (1-4-27-37). In the case of BATMAN, as shown in Figure 5(b), the corresponding path is $(1-5-33-37)$. It is clear from our experiments that OLSR and

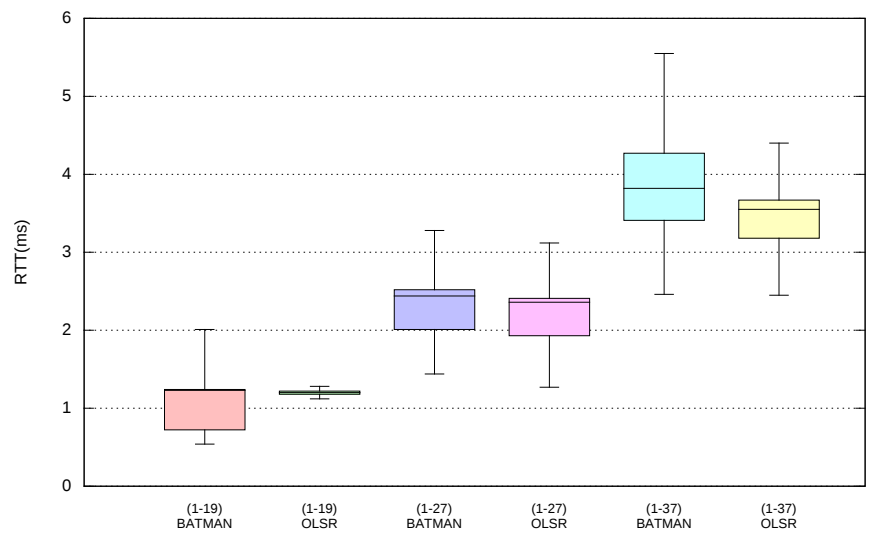

Fig. 6. Distribution of RTT Values without Interference

BATMAN can choose different routes in the exact same network, in some cases with significant differences in path length. This is not surprising, since both protocols vary significantly in regards to their approach to route establishment.

After having considered the basic route establishment of the two WMN routing protocols, we considered their performance in terms of latency and packet delivery ratio (PDR). In order to measure the end-to-end latency of the paths established by both OLSR and BATMAN, we used ping (5000 measurements) between every source destination node pair.

As before, we used paths with node 1 as the source as a representative example, in particular, we considered the following source destination pairs, which represent a 1-hop, 2-hop and 3-hop path respectively: $(1,19),(1,27)$ and $(1,37)$. Figure 6 shows a box and whisker plot of measured RTT values for these 3 paths, both for OLSR and BATMAN. The plot shows the median, the maximum and minimum, as well as the four quartiles.

In Figure 6, the y-axis shows the RTT value of the routes in ms and the $x$-axis shows the source-destination pairs and the corresponding routing protocol. As expected, the RTT value increases with the path length. We see a roughly similar result for both OLSR and BATMAN for the three source destination pairs, despite the fact they chose different paths. This is as expected, since the path length is identical, and the link quality of the corresponding hops is also similar.

We also measured the Packet Delivery Ratio (PDR) for all the network paths. However, due to lack of mobility and significant levels of interference, we did not observe any packet loss, and all paths achieved a PDR value of $100 \%$. To consider a more interesting scenario with packet loss, we looked at a scenario where interference is artificially generated by a node in the R2Lab testbed. We will explore this in the following section.

\section{B. With Interference}

As mentioned before, some nodes on the R2Lab platform are equipped with a USRP device. We used one of these devices to generate noise in the network. In particular, we used the USRP installed on node 11, and with the help of the 


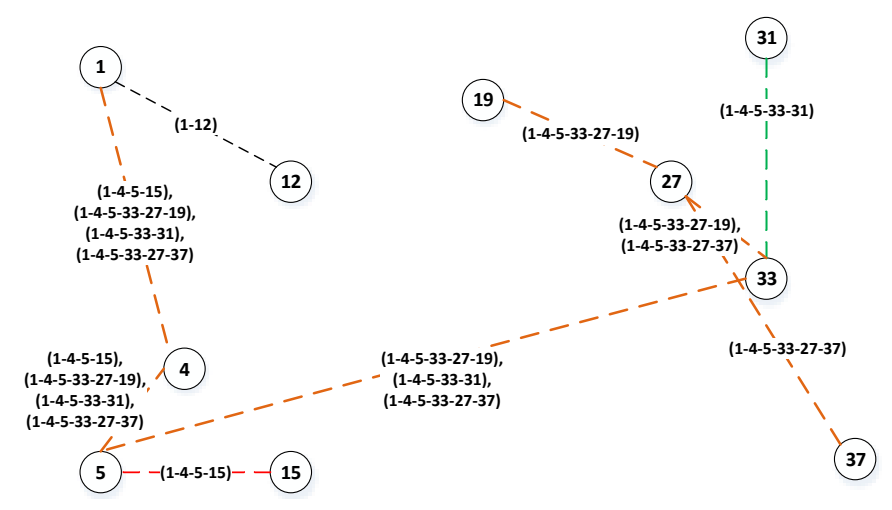

(a) OLSR Routing Protocol

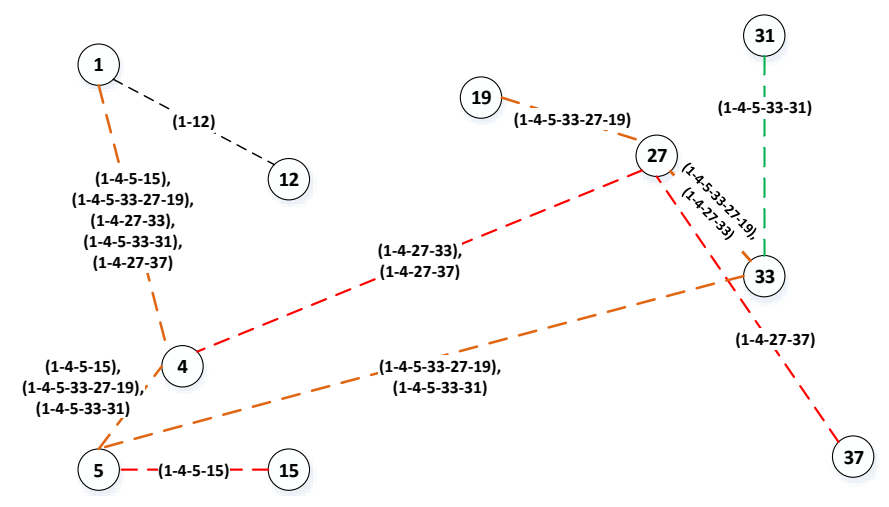

(b) BATMAN Routing Protocol

Fig. 7. Established Route Between Node 1 and Other Nodes After Applying Interference

"uhd_siggen" Linux command we generated Gaussian random noise output with $70 \mathrm{~dB}$ gain.

This noise generating node is located between nodes 1 and 19 and above node 12, as shown in Figure 1. So we excepted these three nodes, and corresponding links, to be affected by the interface.

First, we were interested to see how the interference has an impact on the established routes between our three selected source destination node pairs, i.e. $(1,19),(1,27)$ and $(1,37)$. The result is shown in Figure 7. Figure 7(a) shows the result of OLSR and the routes established by BATMAN are shown in Figure 7(b). The black dashed line represents one-hop paths, blue represents two-hop paths, red represents 3-hop paths, green represents 4-hop paths, and finally orange represents 5hop paths. We can see the injection of interference results in the establishment of longer paths, since both OLSR (with the ETX routing metric) and BATMAN avoid shorter but lower quality paths.

If we consider the path between nodes 1 and 19 for example, we can see that the one-hop path in the scenario without interference has now been replaced with a 5-hop path, going via the following nodes: 1, 4, 5, 33, 27, 19, as shown in Figure 7.

We performed the same experiments to measure path RTT

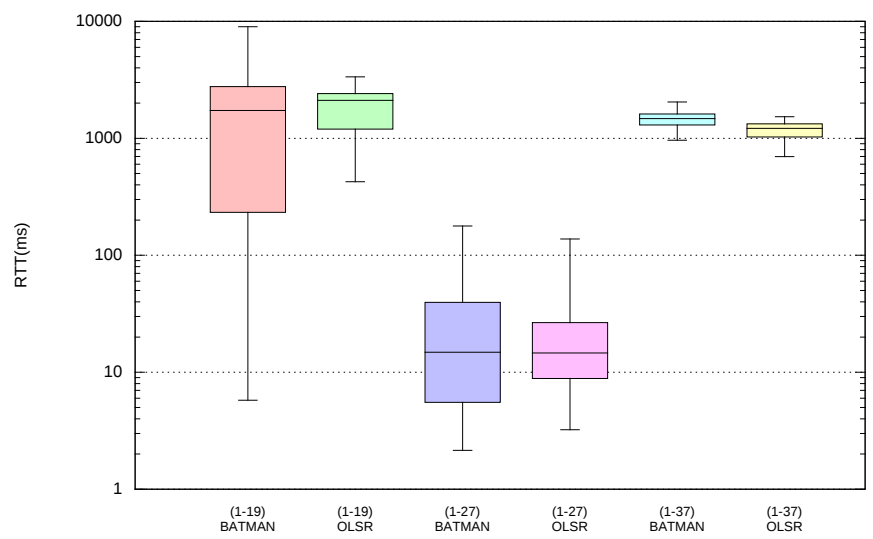

Fig. 8. Distribution of RTT Values with Interference

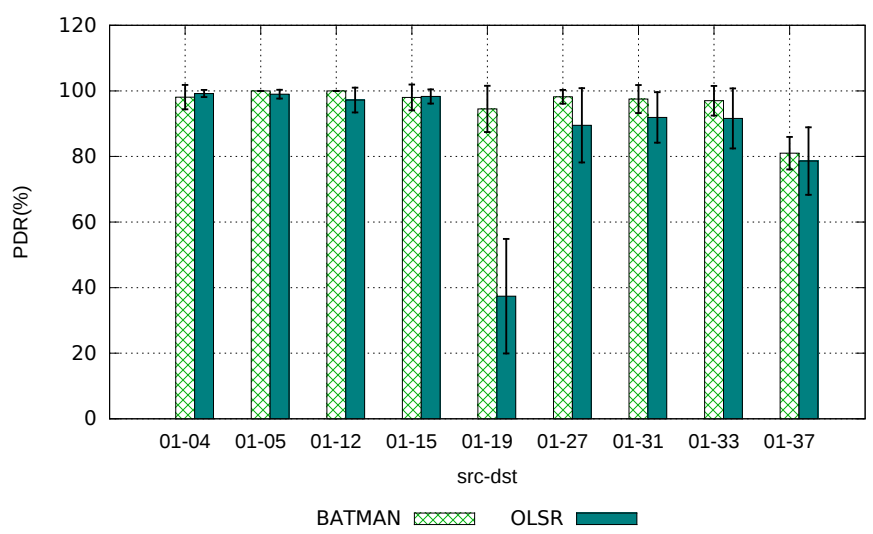

Fig. 9. PDR Value with Interference for Node 1 as Source and Other Nodes as Destinations

and PDR, as we have done in the case without interference. Figure 8 shows the RTT results, considering the paths from node 1 to nodes 19, 27 and 37 respectively, as in our previous scenario without interference. We see a significant increase in RTT values overall, with some values of well above 1000 ms. (Note that the y-axis (RTT) is in logarithmic scale here.) This is due to two main reasons. The first is the significant increase in path length, as discussed above. The second reason is the lower quality of wireless links, which results in a higher number of packet retransmission.

We observe that both OLSR and BATMAN are similarly impacted by the increase in RTT due to interference. Again, this is as expected, since both protocols aim to avoid low quality paths, and therefore establish significantly longer paths.

We also measured the PDR on these paths, as in our previous scenario without interference. Figure 9 shows the results. The graph shows the average of 10 experiment runs, with the $95 \%$ confidence interval.

The $\mathrm{x}$-axis shows the considered source-destination pair. The y-axis shows the PDR value in percent. In contrast to the scenario, where we did not have an interference, we now observe paths with less than $100 \%$ PDR. Overall, BATMAN 
and OLSR perform similarly, with the exception of source destination pair $(1,19)$, where the path chosen by OLSR achieves a PDR of below $40 \%$, while BATMAN achieves a PDR of greater than $90 \%$. This can be explained by the different approaches of considering link/path quality by the two protocols, i.e. OLSR uses link state routing with the ETX metric, while BATMAN does consider path quality based on the best one-hop neighbour. Overall, the achieved results are as expected, and hence validate the R2Lab testbed as a valid platform for wireless multi-hop experiments.

\section{CONCLUSIONS}

We have presented a first experimental validation of the R2Lab wireless testbed platform, in regards to wireless multi hop experiments. In particular, we have performed a basic evaluation of the OLSR and BATMAN WMN routing protocols, in terms of latency and PDR. We considered a scenario without interference, and a scenario where we injected interference, and we observed the impact on the route selection for both protocols. Overall, the obtained results give us great confidence about the suitability of the R2Lab testbed for experiments in Wireless Mesh Networking. Given the separation of the data plane and control plane in the R2Lab architecture, we believe it provides a great potential for experiments for SDN-based WMNs which remains to be explored in our future work.

\section{ACKNOWLEDGMENT}

The authors would like to acknowledge the financial support through an "Australian Government Research Training Program Scholarship" and the University of Queensland Graduate School for the Graduate School International Travel Award (GSITA).

\section{REFERENCES}

[1] R2Lab testbed, INRIA, https://r2lab.inria.fr/. [Online]. Available: https://r2lab.inria.fr/

[2] USRP, ETTUS, https://www.ettus.com/. [Online]. Available: https://www.ettus.com/

[3] inaugural meeting R2Lab testbed, INRIA, https://www.inria.fr/en/centre/sophia/calendar/ r2lab-anechoicchamber-a-heterogeneous-wireless-testbed. [Online]. Available: https://www.inria.fr/en/centre/sophia/calendar/ r2lab-anechoic-chambera-heterogeneous-wireless-testbed

[4] ORBIT, ORBIT, http://www.orbit-lab.org/. [Online]. Available: http://www.orbit-lab.org/

[5] D. Raychaudhuri, I. Seskar, M. Ott, S. Ganu, K. Ramachandran, H. Kremo, R. Siracusa, H. Liu, and M. Singh, "Overview of the orbit radio grid testbed for evaluation of next-generation wireless network protocols," in Wireless Communications and Networking Conference, 2005 IEEE, vol. 3. IEEE, 2005, pp. 1664-1669.

[6] B. White, J. Lepreau, L. Stoller, R. Ricci, S. Guruprasad, M. Newbold, M. Hibler, C. Barb, and A. Joglekar, "An integrated experimental environment for distributed systems and networks," ACM SIGOPS Operating Systems Review, vol. 36, no. SI, pp. 255-270, 2002.

[7] J. Zhou, Z. Ji, M. Varshney, Z. Xu, Y. Yang, M. Marina, and R. Bagrodia, "Whynet: a hybrid testbed for large-scale, heterogeneous and adaptive wireless networks," in Proceedings of the 1st international workshop on Wireless network testbeds, experimental evaluation \& characterization. ACM, 2006, pp. 111-112.

[8] T. Clausen and P. Jacquet, "Optimized link state routing protocol (olsr)," Tech. Rep., 2003, https://tools.ietf.org/html/rfc3626.
[9] A. Neumann, C. Aichele, M. Lindner, and S. Wunderlich, Better Approach To Mobile Ad-hoc Networking (B.A.T.M.A.N.) draft-wunderlich-openmesh-manet-routing00, Available from: "http://www.open-mesh.net/", [31 March 2017], 2008, https://tools.ietf.org/html/draft-wunderlich-openmeshmanet-routing-00. [Online]. Available: https://tools.ietf.org/html/draftwunderlich-openmesh-manet-routing-00

[10] R. Draves, J. Padhye, and B. Zill, "Comparison of routing metrics for static multi-hop wireless networks," in ACM SIGCOMM Computer Communication Review, vol. 34, no. 4. ACM, 2004, pp. 133-144.

[11] M. Abolhasan, B. Hagelstein, and J.-P. Wang, "Real-world performance of current proactive multi-hop mesh protocols," in Communications, 2009. APCC 2009. 15th Asia-Pacific Conference on. IEEE, 2009, pp. 44-47.

[12] Optimized Link State Routing Protocol (OLSR), OLSR, http://www.olsr.org/ mediawiki/index.php/Olsrd_releases. [Online]. Available: http://www.olsr.org/mediawiki/index.php/Olsrd_releases

[13] D. S. De Couto, D. Aguayo, J. Bicket, and R. Morris, "A high-throughput path metric for multi-hop wireless routing," Wireless Networks, vol. 11, no. 4, pp. 419-434, 2005. 